\title{
Altered Functional Network Affects Amyloid and Structural Covariance in Alzheimer's Disease
}

\author{
Ya-Ting Chang $(\mathbb{D}$, Chi-Wei Huang, Wen-Neng Chang, \\ Jun-Jun Lee, and Chiung-Chih Chang (D) \\ Department of Neurology, Kaohsiung Chang Gung Memorial Hospital, Chang Gung University College of Medicine, \\ Kaohsiung 83301, Taiwan \\ Correspondence should be addressed to Chiung-Chih Chang; neur099@cgmh.org.tw
}

Received 9 August 2018; Accepted 15 November 2018; Published 2 December 2018

Academic Editor: Stavros Baloyannis

Copyright (c) 2018 Ya-Ting Chang et al. This is an open access article distributed under the Creative Commons Attribution License, which permits unrestricted use, distribution, and reproduction in any medium, provided the original work is properly cited.

\begin{abstract}
Background. We aimed to investigate how altered intrinsic connectivity networks (ICNs) affect pathologic changes of Alzheimer's disease (AD) at a network-based level. Methods. Thirty normal controls (NCs), 23 patients with AD-mild cognitive impairment (MCI), and 20 patients with AD-dementia were enrolled. We compared the organization of grey matter structural covariance and functional connectivity in ICNs between NCs and all AD patients who were amyloid $\beta(\mathrm{A} \beta)$-positive. We further used seed-based interregional covariance analysis to compare structural and $\mathrm{A} \beta$ plaque covariance in default mode network (DMN) between AD$\mathrm{MCI}$ and AD-dementia groups. Results. The patients with AD had increased functional interregional covariance among the regions of the ICN anchored to dorsal caudate (DC) seeds compared to the NCs. The increased connectivity was associated with extended patterns of reduced $\mathrm{A} \beta$ plaque covariance in the $\mathrm{AD}$-dementia group compared to the $\mathrm{AD}-\mathrm{MCI}$ group within the striatal network anchored to $\mathrm{DC}$ seeds. Patterns of lower $\mathrm{A} \beta$ plaque covariance in the $\mathrm{AD}$-dementia group compared to the $\mathrm{AD}$-MCI group were more extended within the network anchored to DC seeds than within the DMN, which was undergoing functional failure in the patients with AD. Significant decreased structural covariance in the AD-dementia group compared to the AD-MCI group was more extended in the DMN during functional failure. Conclusions. Functional connectivity in ICNs affects the topographic spread of molecular pathologies. The temporal trajectory of pathologic alterations can be well demonstrated by pathologic covariance comparisons between different clinical stages. Pathologic covariance can provide critical support to pathologic interactions at network and molecular levels.
\end{abstract}

\section{Introduction}

The tau propagation theory [1] states that neurofibrillary tangles in Alzheimer's disease (AD) are deposited along intrinsic connectivity networks (ICNs), and interconnected regions with high basal metabolism have been reported to be targets for accelerated pathologic accumulation $[2,3]$. Meanwhile, alterations in ICN dynamics are also believed to change the expanding nature of amyloid $\beta(\mathrm{A} \beta)$ in $\mathrm{AD}[3,4]$.

Healthy individuals have high functional connectivity in the default mode network (DMN) [5]; nodes within this functional network (FN) have exhibited tightly correlated grey matter volumes [4]. This structural covariance map is referred to as a structural network (SN) [4]. Significantly reduced structural covariance in DMN hubs has been observed in the early stage of $\mathrm{AD}[6]$; however reports on the relationships with functional alternations as revealed by resting state function magnetic resonance imaging (rs-fMRI) have been consistent [6].

In vivo amyloid positron emission tomography (PET) has also shown that fibrillar $A \beta$ loads of regions typically involved in the DMN demonstrate high collinearity, the socalled amyloid network (AN) [3]. As the DMN remains a neuroimaging hallmark in the early clinical stage of $\mathrm{AD}[7$, 8], the assessment of functional connectivity and pathologic burden can only be performed at this particular clinical stage [3]. In other words, comparisons of alterations in the SN and AN between different stages of the disease may help to incorporate the concept of ICNs into the spatiotemporal framework of an $\mathrm{AD}$ biomarker model [9].

On functional failure in the DMN, ICNs involved with the striatum [10] have been reported to subsequently exhibit 
increased functional connectivity [11]. Among the striatal networks, the dorsal caudate (DC) and its functional interconnected regions have been shown to be an ideal model to assess cognitive modulation in $\mathrm{AD}$ because of the involvement with heteromodal association cortices [12]. Exploring relationships between $\mathrm{FN}$ and the molecular pathologic networks anchored to DC seeds [12] may help to elucidate the mechanisms by which alterations in ICNs influence the processes facilitating the pathologic spread in $\mathrm{AD}$.

Regarding the pathophysiologic model of AD, molecular pathology deposits along the ICNs [13], patients with $\mathrm{AD}$ have decreased structural covariance among regions of the DMN [6], and progressive network changes in AD [14] are characterized by functional failure in the DMN and a compensatory increased connectivity in other FNs [10, 15]. However, no cross-sectional studies have simultaneously measured AD-associated changes in ANs, SNs, and FNs to directly compare alterations in pathology at a network level.

Seed regions have commonly been used to investigate syndrome-related FNs [4] and SNs [4, 6]. As ICNs have been shown to have a clinical impact $[4,16]$, the aim of this study was to investigate the relationship between seed volume and cognitive performance. Using rs-fMRI and high resolution structural images in florbetapir (18F-AV-45)-positive AD [17], we explored whether the pattern of decreased structural covariance in patients with $\mathrm{AD}$ as compared to normal controls (NCs) was different between the ICNs undergoing functional failure and those undergoing a compensatory increase. We then compared the pattern of $\mathrm{A} \beta$ plaque covariance in patients with $\mathrm{AD}$-dementia and $\mathrm{AD}$-mild cognitive impairment (MCI). We hypothesized that high functional connectivity in each ICN would accelerate the accumulation of fibrillar $\mathrm{A} \beta$ along the same network through the dementia phase of the disease. Therefore, we investigated whether the pattern of decreased $\mathrm{A} \beta$ plaque covariance in patients with $\mathrm{AD}$-dementia, relative to those with $\mathrm{AD}-\mathrm{MCI}$, was more extensive within ICNs undergoing compensatory increase than within those undergoing functional failure.

\section{Methods}

2.1. Inclusion and Exclusion Criteria. Forty-three patients with $\mathrm{AD}-\mathrm{MCI}$ or $\mathrm{AD}$-dementia $[17,18]$ were enrolled from Department of General Neurology of Chang Gung Memorial Hospital from 2011 to 2017. The patients were included based on the consensus of panels comprising neurologists, neuropsychologists, neuroradiologists, and experts in nuclear medicine [19]. A Clinical diagnosis of AD-dementia or ADMCI was based on the International Working Group criteria with positive PET amyloid imaging of $18 \mathrm{~F}-\mathrm{AV}-45$ by visual rating $[17,18]$. Only the patients with a clinical dementia rating $(\mathrm{CDR})$ scores of 1 were included in the $\mathrm{AD}$-dementia group. Patients were included in the AD-MCI group if they had (1) concerns regarding a change in cognition from the patient or an informant, (2) scores on cognitive tests of approximately 1 to 1.5 standard deviations below the education- and age-adjusted NCs in one or more cognitive domains, and (3) preservation of independence in functional abilities without dementia. The exclusion criteria were a clinical history of stroke, modified Hachinski ischemic score > 4 [20], and depression.

A volunteer group of education- and age- matched NCs, who had no underlying neurological or psychiatric disorders, was recruited from outpatient neurological clinics. The Human Ethics Committee of Chang Gung Memorial Hospital approved the study protocol. All of the subjects or their authorized caregivers provided written informed consent.

2.2. Study Design. 18F-AV-45 PET, cognitive testing, and magnetic resonance imaging (MRI) were performed within a 4-week period.

2.3. Image Acquisition. MR studies were conducted at $3 \mathrm{~T}$ using a GE Signa Excite scanner (GE Medical System, Milwaukee, WI). The scanning protocol for 3-dimensional (3D) T1-weighted images was as follows: inversion-recoveryprepared, 3D, spoiled, gradient-recalled acquisition in a steady-state sequence with repetition time/inversion time of $8,600 \mathrm{~ms} / 450 \mathrm{~ms}, 240 \times 240 \mathrm{~mm}$ field of view, and 1 $\mathrm{mm}$ slice thickness. Rs-fMRI scans were performed with the patients' eyes closed using a T2* -weighted echo-planar imaging sequence (TR 2500ms, TE $45 \mathrm{~ms}$, FOV $240 \times 240$ $\mathrm{mm}$, flip angle $10^{\circ}$, thickness $4 \mathrm{~mm}$, and 200 scans of 32 contiguous axial slices) with a total scanning time of 10 minutes per subject.

$18 \mathrm{~F}-\mathrm{AV}-45$ was synthesized at the cyclotron facility of Chang Gung Memorial Hospital. The PET acquisition protocol, optimal scanning time, and image reconstruction were according to a previous study [21].

\subsection{Data Preprocessing}

2.4.1. Structural Volume and PET. 3D $\mathrm{T}_{1}$-weighted MRI and 18F-AV-45 PET data were preprocessed using Statistical Parametric Mapping 12 (SPM12) software (http://www .fil.ion.ucl.ac.uk/spm/) running under MATLAB 7.9 (MathWorks, Natick, MA).

Preprocessing included realignment, segmentation, normalization into standard stereotactic Montreal Neurological Institute (MNI) spaces, and spatial smoothing using a Gaussian Kernel of $5 \mathrm{~mm}$ full-width at half-maximum (FWHM) for T1 and $7 \mathrm{~mm}$ FWHM for PET. Using diffeomorphic anatomical registration via the exponentiated lie algebra approach, related tissue segments were used to create a custom template for both T1 and PET. Using REST version 1.8 [22], the PET images were normalized to white matter regions [23]. 18F-AV-45 standardized uptake value ratio (SUVr) of the seed regions was extracted and used in further analysis. The raw volume of interest (VOI) of the hippocampus and total intracranial volume (TIV) were estimated using surfacebased atlas maps in Computational Anatomy Toolbox 12 in SPM12 [24]. Hippocampal VOI analysis used the TIVadjusted volume.

2.4.2. Rs-fMRI. The first 10 volumes of rs-fMRI were discarded to reduce fluctuations in MRI signals. The preprocessing steps of rs-fMRI included slice time correction, realignment, segmentation, normalization into standard stereotactic 
MNI spaces, and spatial smoothing using a Gaussian Kernel of $6 \mathrm{~mm}$ and resampling to $2 \times 2 \times 2 \mathrm{~mm}^{3}$. Subsequently, functional images were smoothed with $6 \mathrm{~mm}$ FWHM.

For motion artifacts, the following cut-off scores were used: (1) less than $1 \%$ variation in the global average blood oxygen level dependent (BOLD) signal from scan to scan and (2) $0.25 \mathrm{~mm} / \mathrm{TR}$ of frame-wise displacement. Simultaneously, images were detrended and filtered to $0.008 \sim 0.09 \mathrm{~Hz}$. After regressing out the head movement time series, white matter and cerebrospinal fluid signals were extracted from each voxel using an anatomical component-based noise correction method as implemented in CONN functional connectivity toolbox (http://www.nitrc.org/projects/conn) [25, 26]. Seed based individual correlation maps using the CONN toolbox were constructed by extracting the mean resting-state BOLD time course from entorhinal seeds and DC seeds. The significance maps of seed-to-voxel connectivity in all $\mathrm{AD}$ patients as compared to NCs were computed using second-level analysis of relative functional connectivity using a two-sided independent $t$-test (peak-level at uncorrected $p<$ 0.001; cluster-level at false discovery rate [FDR] corrected $p<$ 0.05). The correlation coefficients between the seed regions and all the significant clusters represented the voxel-wised functional connectivity for entorhinal seeds and DC seeds. All the resulting coefficients were converted to normally distributed scores using Fisher's transformation.

2.5. Neurobehavioral Assessments. We used the Mini-Mental Stage Examination (MMSE) [27] to assess general cognitive function, and functional severity was determined using CDR scores. Episodic memory was assessed using the Chinese version of the verbal learning test (CVVLT) [28], and we recorded the four verbal learning trials of a 9-word list with free recall after 10 minutes (CVVLT-10 min) and cued recall (CVVLT-cued) (number of words recalled with cued procedures over four learning trials). The CVVLT-10 min was used to evaluate delayed recall, and CVVLT-cued was used to measure memory under a cued response. Visuospatial function was assessed by visual object and space perception (VOSP) [29] and modified Rey-Osterrieth (mR-O) complex figure copying [30], and executive functions were assessed using Stroop interference [31] and the Trails Making Test parts B (TMT parts B) [32].

2.6. Seed-Based Analyses. Seed-based analyses were performed using two main steps. First, volume and normalized 18F-AV-45 SUVr were extracted from a $4 \mathrm{~mm}$ radius sphere around the bilateral entorhinal $( \pm 25,-9,-28)[6]$ and bilateral DC $( \pm 13,15,9)$ [33] seeds. Second, statistical contrasts were set to identify the voxels reflecting differences between groups in regression slopes for each seed. Differences in the slopes were referred to as the differences in structural and normalized 18F-AV-45 SUVr covariance [6]. Using the threshold of family-wise error corrected $p<0.05$ at the voxel level and uncorrected $p<0.01$ at the cluster level with a cluster size $>100$ voxels, we established the map of voxels that expressed a stronger structural covariance in the NCs compared to all patients with $\mathrm{AD}$. Using the same threshold, we further established the map of voxels that expressed a stronger structural covariance and $\mathrm{A} \beta$ plaque covariance in the patients with AD-MCI compared to those with $\mathrm{AD}$ dementia.

2.7. Statistical Analysis. Clinical data were expressed as mean \pm standard deviation. Student's $t$-test was used to compare continuous variables between the AD-MCI, ADdementia, and NCs groups, with post hoc Bonferroni correction. Statistical significance was set at $p<0.05$. All statistical analyses were conducted using SPSS software (SPSS version 22 for Windows ${ }^{\circledR}$, SPSS Inc., Chicago, IL).

\section{Results}

3.1. Comparisons of Clinical and Pathologic Data. The demographic data are shown in Table 1. Patients with AD-MCI had significantly lower episodic memory scores and hippocampal volume than the NCs $(p<0.05)$. There were no significant differences between the AD-MCI and NCs groups in other cognitive scores $(p>0.05)$. The $\mathrm{AD}$-dementia group had lower episodic memory scores, hippocampal volume, and all the other cognitive scores compared to the AD-MCI and NCs groups $(p<0.05)$.

3.2. Importance of Seed Volume and Its Relationship with Memory Performance. There was a significant positive correlation between right entorhinal seed volume and episodic memory scores including delayed recall and memory under a cued response ( $p<0.05$; Table 2$)$; however the right DC seed volume was only correlated with memory under a cued response ( $p<0.05$; Table 2$)$. Regarding other cognitive functions, the right entorhinal seed volume was only associated with Stroop interference scores $(p=0.01$; Table 2$)$, whereas bilateral DC seeds were correlated with all executive function scores and mR-O complex figure copying scores $(p<0.05$; Table 2). Since cognitive decline has been supposed to be loosely associated with the magnitude of $\mathrm{A} \beta$ plaque load [9], we did not use seed 18F-AV-45 SUVr to correlate with memory performances.

This exploratory analysis served to better establish the clinical impacts of entorhinal and DC seed volumes anchored by syndrome-related SN [4]. We next verified our assumption that alteration in FNs anchored to entorhinal and DC seeds would affect the changes in SN anchored to entorhinal and DC seeds in all patients with AD as compared to NCs.

\subsection{Differences in SNs Anchored to Entorhinal and DC Seeds}

3.3.1. Decreased Structural Covariance in All Patients with $A D$ Compared to the NCs. Within the SN anchored to the right entorhinal seed, all patients with $\mathrm{AD}$ exhibited lower structural covariance than NCs among the regions involved in the DMN (total: 825 voxels), while within the $\mathrm{SN}$ anchored to the right DC seed, the comparison of structural covariance between all patients with AD and NCs did not reach the pre-established statistical threshold (Figure 1; Supplementary Table 1 in Supplementary Data). 
TABLE 1: Demographic data of the study participants.

\begin{tabular}{|c|c|c|c|}
\hline & Normal controls & AD-MCI & AD-dementia \\
\hline Male/Female & $17 / 13$ & $12 / 11$ & $11 / 9$ \\
\hline Age (years) & $65.9 \pm 5.6$ & $71.4 \pm 8.1$ & $70.0 \pm 6.7$ \\
\hline Education (years) & $9.0 \pm 4.8$ & $10.8 \pm 4.1$ & $7.7 \pm 5.4^{\mathrm{b}}$ \\
\hline \multicolumn{4}{|l|}{ Cognitive test score } \\
\hline Mini-mental state examination & $27.1 \pm 3.2$ & $26.3 \pm 2.3$ & $19.0 \pm 4.7^{\mathrm{ab}}$ \\
\hline \multicolumn{4}{|l|}{ Episodic memory scores } \\
\hline CVVLT-10 min & $7.2 \pm 1.5$ & $5.87 \pm 2.4^{\mathrm{a}}$ & $1.5 \pm 2.1^{\mathrm{ab}}$ \\
\hline CVVLT-cued & $7.3 \pm 1.3$ & $6.2 \pm 2.2^{\mathrm{a}}$ & $1.9 \pm 2.1^{\mathrm{ab}}$ \\
\hline \multicolumn{4}{|l|}{ Executive function scores } \\
\hline Trail Making Test parts B & $11.7 \pm 4.3$ & $11.7 \pm 3.2$ & $5.3 \pm 4.8^{\mathrm{ab}}$ \\
\hline Stroop interference & $35.3 \pm 12.4$ & $28.7 \pm 15.7$ & $18.1 \pm 12.7^{\mathrm{ab}}$ \\
\hline \multicolumn{4}{|l|}{ Visuospatial function scores } \\
\hline VOSP & $8.1 \pm 2.4$ & $8.3 \pm 2.0$ & $4.6 \pm 3.5^{\mathrm{ab}}$ \\
\hline mR-O complex figure copying & $16.3 \pm 2.2$ & $16.7 \pm 0.9$ & $13.8 \pm 6.0^{\mathrm{ab}}$ \\
\hline \multicolumn{4}{|l|}{ TIV adjusted VOI volume $* 10^{-3}$} \\
\hline Right hippocampus & $1.5 \pm 0.1$ & $1.4 \pm 0.2^{\mathrm{a}}$ & $1.1 \pm 0.2^{\mathrm{ab}}$ \\
\hline Left hippocampus & $1.4 \pm 0.1$ & $1.4 \pm 0.2^{\mathrm{a}}$ & $1.1 \pm 0.2^{\mathrm{ab}}$ \\
\hline
\end{tabular}

Values with mean \pm standard deviation. ${ }^{\mathrm{a}} p<0.05$, significant difference compared to normal controls using the independent $t$-test. ${ }^{\mathrm{b}} p<0.05$, significant difference compared to AD-MCI using the independent $t$-test in AD-dementia. AD, Alzheimer's disease; CVVLT-10 min, Chinese version of the Verbal Learning Test with 10 minutes' interval recall; CVVLT-cued, Chinese version of the Verbal Learning Test-cued recall; MCI, mild cognitive impairment; mR-O, modified Rey-Osterrieth; TIV, total intracranial volume; VOI, volume of interest; VOSP, visual object and space perception.

TABLE 2: Correlation between scores of memory performance tests and seed volumes.

\begin{tabular}{|c|c|c|c|c|}
\hline Seed & Right entorhinal & Left entorhinal & Right DC & Left DC \\
\hline Dependent variable & $\begin{array}{c}\rho \text { value } \\
(\rho \text { value })\end{array}$ & $\begin{array}{c}\rho \text { value } \\
(p \text { value })\end{array}$ & $\begin{array}{c}\rho \text { value } \\
(p \text { value })\end{array}$ & $\begin{array}{c}\rho \text { value } \\
(p \text { value })\end{array}$ \\
\hline \multicolumn{5}{|l|}{ Episodic memory scores } \\
\hline CVVLT-10min & $\begin{array}{c}0.356 \\
(0.002)\end{array}$ & $\begin{array}{c}0.119 \\
(0.319)\end{array}$ & $\begin{array}{c}0.186 \\
(0.118)\end{array}$ & $\begin{array}{c}0.110 \\
(0.385)\end{array}$ \\
\hline CVVLT-cued & $\begin{array}{c}0.301 \\
(0.010)\end{array}$ & $\begin{array}{c}0.117 \\
(0.326) \\
\end{array}$ & $\begin{array}{c}0.285 \\
(0.015) \\
\end{array}$ & $\begin{array}{c}0.185 \\
(0.119) \\
\end{array}$ \\
\hline \multicolumn{5}{|l|}{ Executive function scores } \\
\hline Trail Making Test parts B & $\begin{array}{c}0.129 \\
(0.280)\end{array}$ & $\begin{array}{c}0.096 \\
(0.421)\end{array}$ & $\begin{array}{c}0.239 \\
(0.043)\end{array}$ & $\begin{array}{c}0.233 \\
(0.049)\end{array}$ \\
\hline Stroop interference & $\begin{array}{c}0.302 \\
(0.010)\end{array}$ & $\begin{array}{c}0.215 \\
(0.069)\end{array}$ & $\begin{array}{c}0.253 \\
(0.032)\end{array}$ & $\begin{array}{c}0.363 \\
(0.002)\end{array}$ \\
\hline \multicolumn{5}{|l|}{ Visuospatial function scores } \\
\hline VOSP & $\begin{array}{c}0.074 \\
(0.539)\end{array}$ & $\begin{array}{c}-0.07 \\
(0.560)\end{array}$ & $\begin{array}{c}0.126 \\
(0.291)\end{array}$ & $\begin{array}{c}0.070 \\
(0.560)\end{array}$ \\
\hline mR-O complex figure copying & $\begin{array}{c}0.141 \\
(0.236)\end{array}$ & $\begin{array}{l}-0.020 \\
(0.867)\end{array}$ & $\begin{array}{c}0.314 \\
(0.007)\end{array}$ & $\begin{array}{c}0.283 \\
(0.016)\end{array}$ \\
\hline
\end{tabular}

Partial correlations controlled for diagnosis. Values with $\rho$ value ( $p$ value). Bold font indicates statistical significance at $p<0.05$. CVVLT-10 min, Chinese version of the Verbal Learning Test with 10 minutes' interval recall; CVVLT-cued, Chinese version of the Verbal Learning Test-cued recall; DC, dorsal caudate; mR-O, modified Rey-Osterrieth; VOSP, visual object and space perception.

Within the SN anchored to the left entorhinal seed, all patients with $\mathrm{AD}$ exhibited lower structural covariance than the NCs in the regions of the right supramarginal gyrus (total: 150 voxels), while within the SN anchored to the left DC seed, the comparison of structural covariance between all patients with $\mathrm{AD}$ and NCs did not reach the pre-established statistical threshold (Figure 1; Supplementary Table 2 in Supplementary Data). 

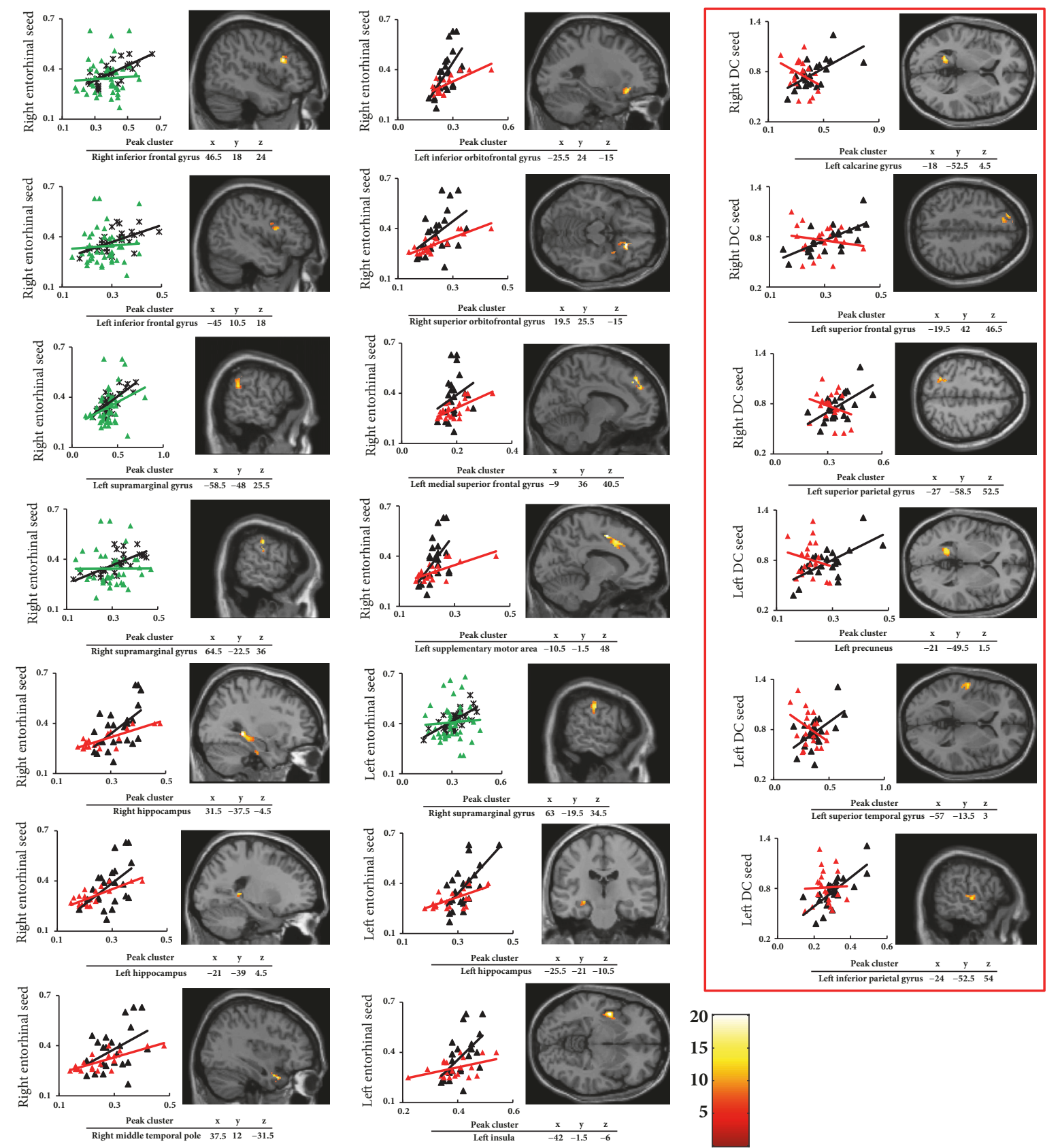

$$
\begin{array}{lll}
*-\text { Normal controls } & \boldsymbol{\Delta}-\text { AD-MCI group } \\
\boldsymbol{A} \text { - All patients with } \mathrm{AD} & \boldsymbol{\Delta}-\text { AD-dementia group }
\end{array}
$$

FIGURE 1: The peak clusters that showed lower structural covariance anchored to bilateral entorhinal seeds $( \pm 25,-9,-28)$ and bilateral DC seeds (within the red square) $( \pm 13,15,9)$ in all of the patients with $\mathrm{AD}$ compared to the normal controls and the $\mathrm{AD}$-dementia group compared to the $\mathrm{AD}-\mathrm{MCI}$ group. Correlations between GM volumes extracted from a sphere of $4 \mathrm{~mm}$ in radius surrounding bilateral entorhinal seeds/bilateral DC seeds and each $4 \mathrm{~mm}$ radius sphere around all peak voxels expressing lower structural covariance in all of the patients with AD compared to the normal controls and the $\mathrm{AD}$-dementia group compared with the $\mathrm{AD}-\mathrm{MCI}$ group. AD, Alzheimer's disease; DC, dorsal caudate; GM, grey matter; MCI, mild cognitive impairment.

3.3.2. Decreased Structural Covariance in the AD-Dementia Group Compared to the AD-MCI Group. Within the SN anchored to the right entorhinal seed (Figure 1), the ADdementia group had decreased structural covariance as compared to the AD-MCI group among the regions involved in the DMN (total: 2106 voxels; Supplementary Table 1 in Supplementary Data), while within the SN anchored to the right DC seed (Figure 1), lower structural covariance was less extended (total: 634 voxels; Supplementary Table 1 in Supplementary Data). 

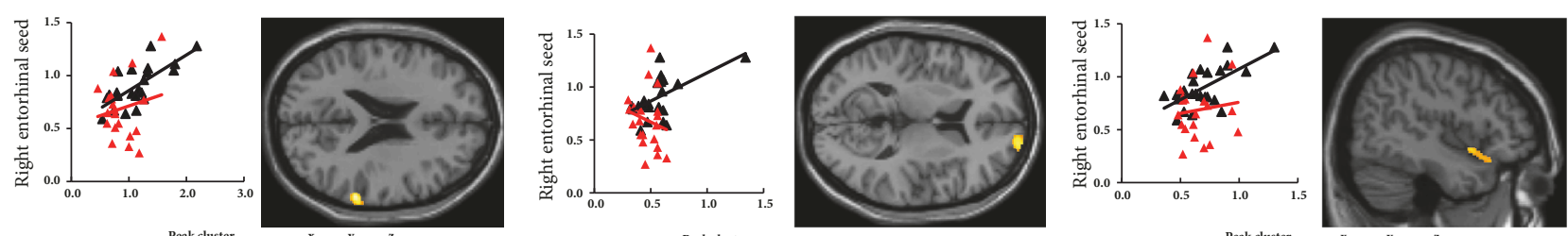

\begin{tabular}{cccc} 
Peak cluster & $\mathrm{x}$ & $\mathrm{y}$ & $\mathrm{z}$ \\
\hline Right superior temporal gyrus & 63 & -37.5 & 22.5
\end{tabular}
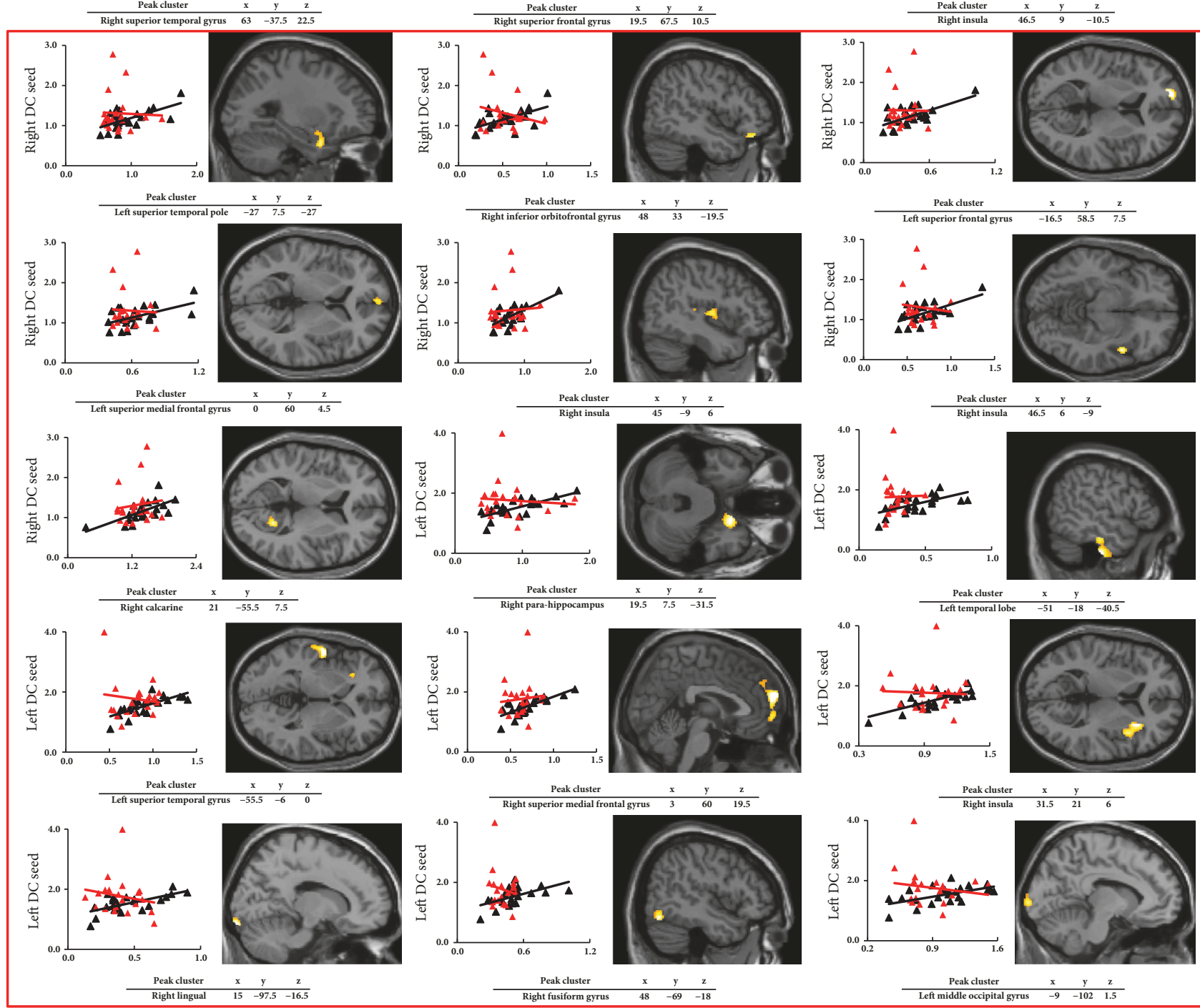

$\Delta-$ AD-MCI group

$\Delta-$ AD-dementia group

Figure 2: The peak clusters that showed lower A $\beta$ plaque covariance anchored to bilateral entorhinal seeds $( \pm 25,-9,-28)$ and bilateral DC seeds (within the red square) $( \pm 13,15,9)$ in the $\mathrm{AD}$-dementia group compared to the AD-MCI group. Correlations between $18 \mathrm{~F}$-AV- $45 \mathrm{SUVr}$ extracted from a sphere of $4 \mathrm{~mm}$ in radius surrounding bilateral entorhinal seeds/bilateral DC seeds and each 4 mm radius sphere around all peak voxels expressing lower $\mathrm{A} \beta$ plaque covariance in the $\mathrm{AD}$-dementia group compared to the $\mathrm{AD}-\mathrm{MCI}$ group. $18 \mathrm{~F}-\mathrm{AV}-45$, florbetapir; $\mathrm{A} \beta$, amyloid $\beta$; AD, Alzheimer's disease; DC, dorsal caudate; MCI, mild cognitive impairment; SUVr, standardized uptake value ratio.

Within the SN anchored to the left entorhinal seed (Figure 1), the $\mathrm{AD}$-dementia group had decreased structural covariance as compared to the $\mathrm{AD}-\mathrm{MCI}$ group in the regions of left hippocampus and left insula (total: 879 voxels; Supplementary Table 2 in Supplementary Data), while within the SN anchored to the left DC seed (Figure 1), lower structural covariance was less extended (total: 560 voxels; Supplementary Table 2 in Supplementary Data).
3.4. Decreased A $\beta$ Plaque Covariance in the AD-Dementia Group Compared to the AD-MCI Groups. Within AN anchored to the entorhinal seeds (Figure 2), the AD-dementia group had lower $\mathrm{A} \beta$ plaque covariance than the $\mathrm{AD}-\mathrm{MCI}$ group in the regions of the right superior temporal and frontal gyri and right insula (total: 844 voxels; Supplementary Table 3 in Supplementary Data), while within ANs anchored to the DC seeds (Figure 2), lower $\mathrm{A} \beta$ plaque covariance was 


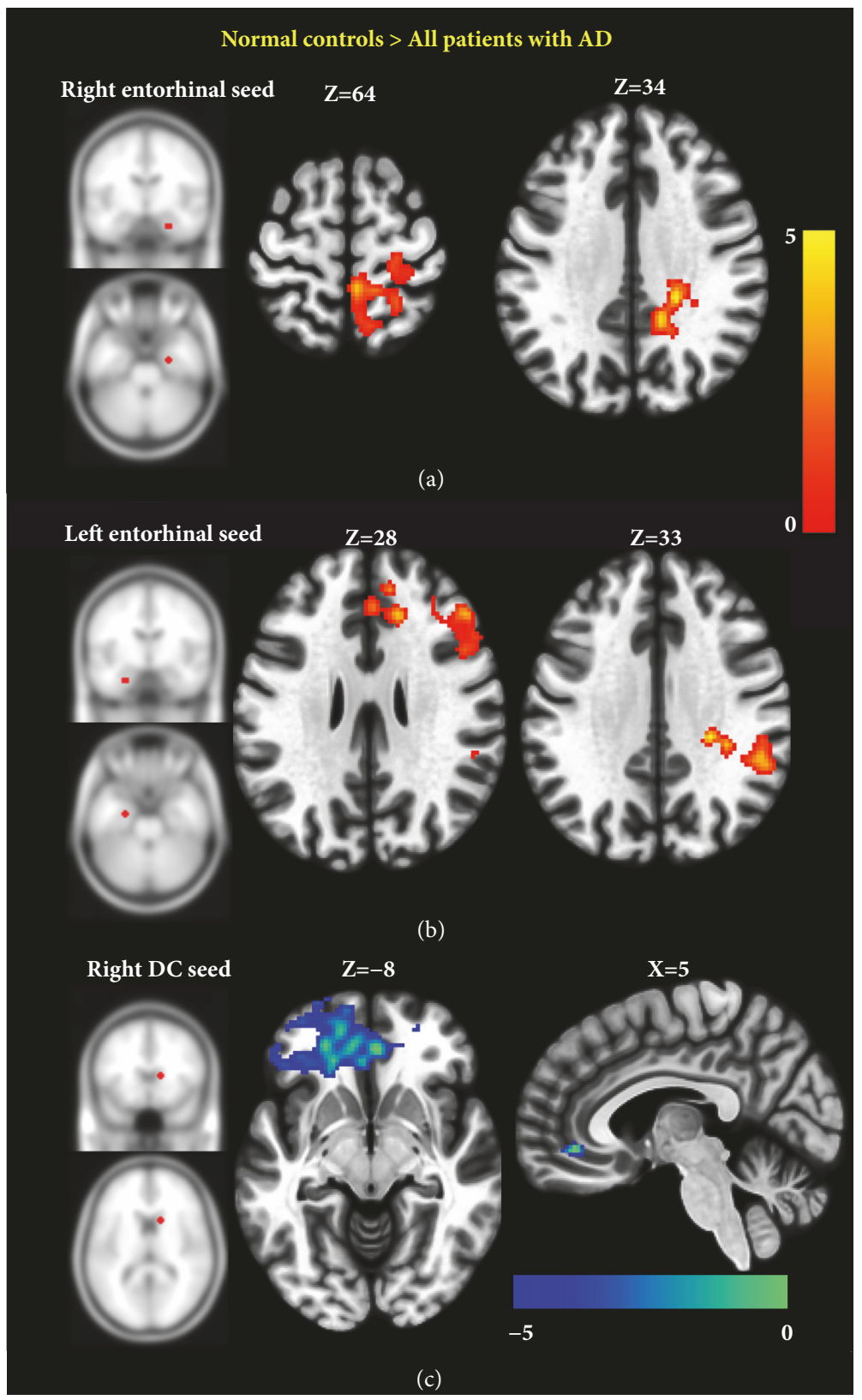

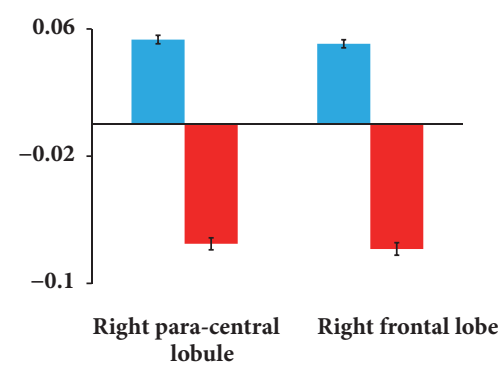

(d)

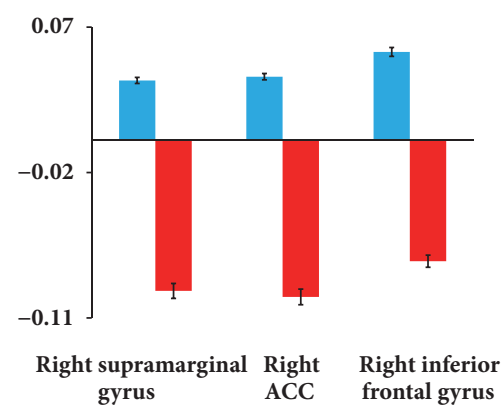

(e)

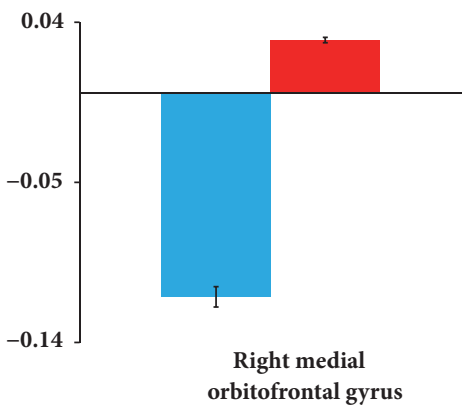

(f)

Normal controls

- All patients with $\mathrm{AD}$

FIGURE 3: Seed-to-voxel analysis in functional networks. Left panels, maps of significant clusters showing functional connectivity with the seed regions in the right entorhinal seed (a), left entorhinal seed (b), and right DC seed (c). Red-yellow color indicates clusters demonstrating reduced functional connectivity with the seed regions in all of the patients with AD relative to the NCs; blue-white color indicates clusters demonstrating increased connectivity. Right panels, mean Fisher's transformed correlation coefficients indicating relative functional connectivity with seed regions in the right entorhinal seed (d), left entorhinal seed (e), and right dorsal caudate seed (f) for each significant cluster in all of the patients with AD and the NCs. ACC, anterior cingulate cortex; AD, Alzheimer's disease; DC, dorsal caudate; NCs, normal controls.

more extended (total: 11268 voxels; Supplementary Table 3 in Supplementary Data).

\subsection{Difference in FN Anchored to Entorhinal and DC Seeds}

3.5.1. Seed-to-Voxel Analysis in All Patients with $A D$ as Compared to the NCs. Within FNs anchored to entorhinal seeds, all patients with $\mathrm{AD}$ had significantly lower functional interregional covariance than NCs in the regions of right paracentral lobule, right frontal lobe, right supramarginal gyrus, right anterior cingulate cortex, and right inferior frontal gyrus (Figures 3(a) and 3(b)).

Within FNs anchored to DC seeds, all patients with AD did not exhibit reduced functional interregional covariance as compared to NCs. However all patients with AD had higher functional interregional covariance than NCs between the 
right DC seed and right medial orbitofrontal gyrus (Figure 3(c)). Figures 3(d)-3(e) show the histograms illustrating relative functional connectivity between seed regions and significant clusters. Table 3 shows T-maxima, MNI coordinate, $P$ value (FDR corrected), and size of each significant cluster in contiguous voxels. All patients with $\mathrm{AD}$ did not exhibit increased functional interregional covariance as compared to NCs within FN anchored to bilateral entorhinal and left DC seeds.

\section{Discussion}

4.1. Main Findings. The present study aimed to elucidate the patterns of $\mathrm{AD}$ biomarkers in the $\mathrm{AD}-\mathrm{MCI}$ and $\mathrm{AD}$ dementia groups by characterizing the changes in ANs, SNs, and FNs. There were three major findings. First, as entorhinal and DC seeds were anchored by syndrome-related ICNs [4], both seed volumes had clinical impacts. Second, all patients with $\mathrm{AD}$ exhibited reduced structural covariance and functional interregional covariance in networks anchored to entorhinal seeds, whereas within networks anchored to DC seeds, these patients with $\mathrm{AD}$ did not show any decrease in structural covariance or functional connectivity. Third, the increased connectivity within FN anchored to the right DC seed in all patients with $\mathrm{AD}$ as compared to NCs suggested a compensatory phenomenon within the striatal network as the disease progressed. Regarding network anchored to DC seeds, as the AD-dementia group had lower structural covariance and $\mathrm{A} \beta$ plaque covariance than the $\mathrm{AD}-\mathrm{MCI}$ group, the decreased covariance exhibited a more extended pattern in the ANs anchored to DC seeds than in the SNs anchored to DC seeds.

4.2. FNs and SNs in DMN and Striatal Network. Distinct clinical syndromes have been associated with dissociable SNs [4]. As clinical features result from diverse network-based pathologic distributions, we first evaluated the clinical impact of seed volumes in the present study. AD typically follows a temporally stereotypical pattern of cognitive impairment, beginning first with episodic memory deficits, followed by dysfunction in diverse cognitive faculties including visuospatial or executive functions [34-36]. Regarding episodic memory function, the entorhinal cortex affects memory encoding, consolidation, and retrieval via its connectivity with the hippocampus and various association cortical regions [37], whereas memory under a cued response depends mostly on the DC [38]. Consistent with these observations, the right DC seed volume in the present study was shown to be specifically associated with memory under a cued response, and the entorhinal seed volume was shown to be involved with various episodic memory functions. We also observed that the DC seed volumes were associated with more widespread cognitive functions including visuospatial and executive performance. DC atrophy and its decreased connectivity with frontal cortices have been linked to executive dysfunction [39]. Although no previous studies have reported an association between DC volume and visuospatial function, lower DC dopamine levels have been associated with greater dysfunction in visuospatial skills [40]. As functional connectivity in striatal networks anchored to DC seeds has been shown to be lower in later disease stages [11], impairment of the DC seed volume-related cognitive function in our data may have occurred in later stages of $\mathrm{AD}$ as a result of striatal network disconnection. This is generally consistent with the literature showing that dysfunction in cognitive processes other than memory temporally follows memory deficits [34-36]. The clinical-pathological relationships of each seed volume suggest that network degeneration also had a clinical impact.

Regarding SNs and FNs, all patients with AD exhibited reduced structural covariance and functional interregional covariance between right entorhinal seed and the regions of frontal lobes and between left entorhinal seed and the regions of supramarginal gyri as compared to NCs. In addition, all patients with $\mathrm{AD}$, relative to NCs, did not demonstrate reduced structural covariance or functional interregional covariance within the network anchored to DC seeds. Corresponding patterns, rather than completely overlap, between altered SN and FN were detected. Some discrepancies may be attributed to the complex mechanisms underlying anatomical and functional interregional covariance, such as developmental, genetic, and environmental factors [41].

The pathologic trajectory of AD suggests that structural topological disorganization of the brain occurs after the stages of accelerated NFT deposition [9]. The cascading network process in $\mathrm{AD}$ proposes that functional connectivity overload is followed by functional failure in DMN during the preclinical stage of AD [14]. The acceleration of NFT accumulation has been attributed to high basal metabolism in the interconnected regions [4] involved in the overloaded DMN [14]. NFT deposition along the DMN has been associated with $\mathrm{FN}$ overload in preclinical stage of $\mathrm{AD}$ [14]; however how alterations in FNs affect anatomic atrophy remains elusive. Regarding temporal evolution, acceleration of NFT accumulation precedes the acceleration of atrophy rates in some brain regions [9]. Such atrophy has been shown to be associated with decreased structural covariance among the hubs of DMN in early clinical stage of AD [6]. Our observations are generally consistent with the literatures $[6,9]$ and expand some of the previous findings. Our study indicates that the decreased structural covariance in DMN accelerates upon functional failure in the DMN and that network failure in both SN and FN occurs after the FN overload-related NFTs deposition. The direct corresponding patterns between the reduction in structural covariance and functional failure within DMN suggest a FN failure-associated SN disruption in early clinical stage of AD.

Within the patients with early clinical stage of $\mathrm{AD}$ presenting with $\mathrm{A} \beta$ plaques, decreased structural covariance in SNs anchored to DC seeds exhibited restricted patterns as the disease progressed. Meanwhile, these AD patients had a compensatory increase in functional connectivity in $\mathrm{FN}$ anchored to right DC seed as compared to NCs. With regard to the findings of high interregional covariance within FNs anchored to DC seeds, such increased metabolic demands may be associated with accelerated NFT propagation along the hubs involved in the striatal network [4]. A more 


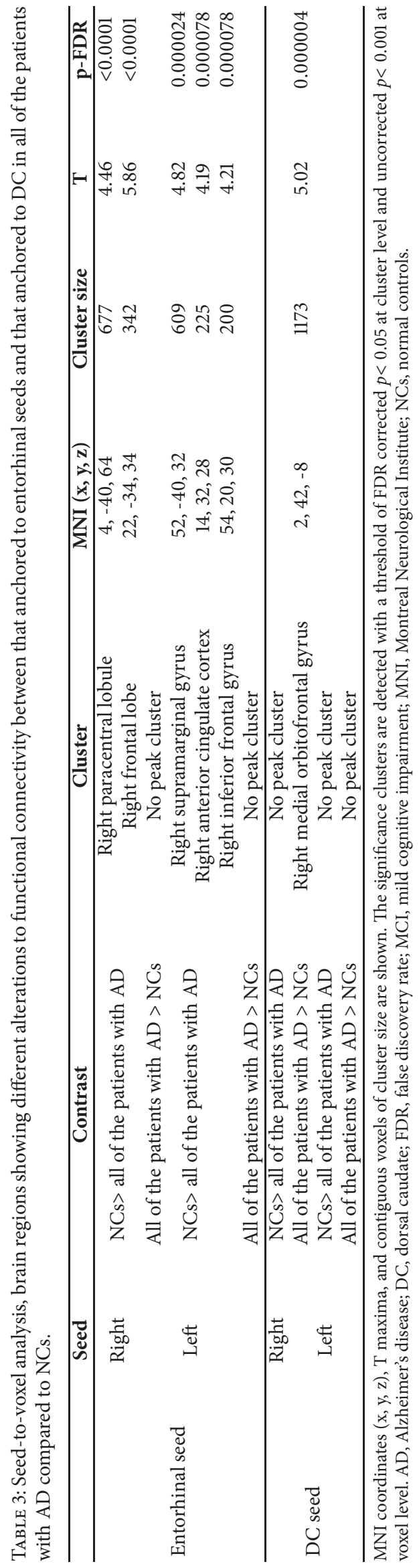


pronounced alteration in $\mathrm{SN}$ anchored to $\mathrm{DC}$ seeds may be prominent in the later clinical stage of $\mathrm{AD}$.

Functional failure in the DMN was associated with an extended pattern of reduced structural covariance in $\mathrm{SN}$ anchored to entorhinal seeds, while the increased connectivity in FNs anchored to DC seeds was associated with a restricted pattern of decreased structural covariance within $\mathrm{SN}$ anchored to DC. The patterns of decreased structural covariance in $\mathrm{AD}$ patients as the disease progresses are generally tracking the network alteration in ICNs as revealed by fMRI.

4.3. FNs and ANs in the DMN and Striatal Network. The deposition of $\mathrm{A} \beta$ plaque has been associated with high excitatory synaptic activity in the interconnected regions of ICNs [3]. It has been suggested that this high basal metabolism triggers downstream A $\beta$ plaque deposition events [42]. Consistent with previous studies, as all of our patients with $\mathrm{AD}$ had a compensatory increased connectivity within FNs anchored to $\mathrm{DC}$ seeds, the $\mathrm{AD}$-dementia group exhibited an extended pattern of reduced $\mathrm{A} \beta$ plaque covariance in $\mathrm{ANs}$ anchored to DC seeds. We also demonstrated a steeper slope of accelerated $\mathrm{A} \beta$ plaque accumulation within the striatal network as the disease progressed in the stage of mild ADdementia.

In contrast to the findings regarding the striatal network, the pattern in the DMN revealed a plateau phenomenon of $\mathrm{A} \beta$ plaque deposition within $\mathrm{AN}$ anchored to entorhinal seeds. Other studies have shown that $\mathrm{A} \beta$ plaque accumulation along the regions involved in the $\mathrm{DMN}$ is only apparent in the preclinical stage of $\mathrm{AD}[3,43]$. The curve representing $\mathrm{A} \beta$ plaque deposition has been reported to show a sigmoidcurved shape and to reach a plateau in the early clinical stage of $\mathrm{AD}[9,44,45]$. The observation that the $\mathrm{AD}$ dementia group had a restricted pattern of reduced $\mathrm{A} \beta$ plaque covariance in AN anchored to entorhinal seed is generally consistent with the theoretic model of pathologic change in $\mathrm{AD}$ [9]. We further demonstrated that such decelerated $\mathrm{A} \beta$ plaque accumulation along the hubs involved in $\mathrm{DMN}$ became apparent on DMN disconnection in the clinical stage of mild AD-dementia.

Functional failure in the DMN was associated with a restricted pattern of reduced $\mathrm{A} \beta$ plaque covariance in ANs anchored to entorhinal seeds, while the increased connectivity in FNs anchored to DC seeds was associated with an extensive pattern of decreased $\mathrm{A} \beta$ plaque covariance within ANs anchored to DC seeds. ICNs-associated spatial distribution should be considered when demonstrating temporal evolution of the pathologic changes [9].

4.4. FNs within DMN and Striatal Network. Mechanisms underlying the changes in FNs anchored to DC seeds in AD are less well-established than those underlying the DMN. However, studies on Parkinson's disease (PD) have emphasized the critical role of the striatal network in cognitive impairment [46]. Decreased functional connectivity between DC seeds and regions including the lateral prefrontal gyrus and posterior parietal cortex has been correlated with executive dysfunction $[47,48]$. Pathologically, the PD dementiaassociated functional failure in the striatal network has been attributed to $\mathrm{A} \beta$ plaque deposition in the $\mathrm{DC}$ [49]. As $\mathrm{A} \beta$ plaque deposition in the striatum has been observed in the later clinical stage of $\mathrm{AD}$ [50], functional failure in striatal network has been postulated to be less pronounced in the early clinical stage.

As functional connectivity declines in the DMN [5], increasing connectivity in other ICNs plays a compensatory role until reaching a functional overload, which subsequently leads to a network-based functional decline in the overloaded ICNs [10]. In agreement with this observation, all of our patients with $\mathrm{AD}$ had failure in FNs anchored to entorhinal seed as compared to the NCs, with simultaneous increased functional connectivity in FNs anchored to DC seeds. Across the entire AD spectrum, the shifting in systems-level ICNs has been referred to as cascading network failure $[14,15]$. Striatal network could be one of the ICNs which plays a compensatory role when DMN undergoes functional failure [42].

\section{Limitations}

This study has several limitations. First, there are limitations inherent to its cross-sectional design, and follow-up studies investigating how different large-scale ICNs affect pathologic evolution across a broader AD spectrum are needed. Second, we only characterized the pathologic and functional patterns of the DMN anchored to entorhinal seeds. Future studies investigating the progression of structural covariance and $\mathrm{A} \beta$ plaque covariance in subsystems of the DMN and striatal networks may help clarify the role of each FN and network-based spatial pathologic changes. Third, the clinical use with $18 \mathrm{~F}-\mathrm{AV}-45$ PET with regard to networks of $\mathrm{A} \beta$ plaques requires further pathologic investigations. However, the regional hierarchy of amyloid deposition signals shown in 18F-AV-45 PET suggests its role in in vivo staging of the progression of amyloid pathology [51].

\section{Conclusions}

We propose that alterations in FNs may affect changes in SNs and ANs. Our model suggested that the DMN fails during the development of $\mathrm{AD}$-dementia and that processing burden is then shifted to striatal network. The accelerated molecular pathologic changes in the regions involved in the ICNs were attributed to increased functional connectivity in the corresponding ICNs. The compensatory increased functional connectivity within the striatal network was associated with acceleration in the decreased $\mathrm{A} \beta$ plaque covariance within ANs anchored to DC seeds. Accelerated deposition of NFTs attributed to high functional connectivity in the DMN was followed by atrophy, and SN anchored to entorhinal seeds exhibited acceleration in the reduced structural covariance as the DMN underwent functional failure. The temporal trajectory of pathologic change can be well demonstrated in pathologic covariance among different regions involved in 
ICNs. Pathologic covariance provides critical support to the pathologic interactions at network and molecular level.

\section{Data Availability}

The datasets used and/or analyzed during the current study are available from the corresponding author on reasonable request.

\section{Ethical Approval}

The study was approved by Chang Gung Memorial Hospital's Institutional Review Committee on Human Research.

\section{Consent}

All of the participants and their authorized caregivers provided written informed consent.

\section{Conflicts of Interest}

The authors declare that they have no conflicts of interest.

\section{Authors' Contributions}

Ya-Ting Chang participated in the design of the study, drafted the manuscript, and performed the statistical analysis. ChiWei Huang, Jun-Jun Lee, and Wen-Neng Chang participated in the sequence alignment and clinical evaluation of patients. Chi-Wei Huang and Chiung-Chih Chang conceived the study, participated in its design and coordination, and helped draft the manuscript. All authors read and approved the final manuscript.

\section{Acknowledgments}

This work was supported by grants CMRPG8C0571 and CMRPG8D0771 from Chang Gung Memorial Hospital and 104-2314-B-182A-026-MY2 from the National Science Council to Chiung-Chih Chang. Funding bodies contributed to collection and interpretation of data.

\section{Supplementary Materials}

Supplementary Table 1: group difference in structural network anchored to the right entorhinal and right DC seeds. Supplementary Table 2: group difference in structural network anchored to the left entorhinal and left DC seeds. Supplementary Table 3: decreased $\beta$-amyloid plaque covariance in $\mathrm{AD}$-dementia compared with $\mathrm{AD}-\mathrm{MCI}$ in amyloid network anchored to the bilateral entorhinal and DC seeds. (Supplementary Materials)

\section{References}

[1] A. de Calignon, M. Polydoro, M. Suárez-Calvet et al., "Propagation of tau pathology in a model of early Alzheimer's disease," Neuron, vol. 73, no. 4, pp. 685-697, 2012.
[2] J. J. Palop and L. Mucke, "Amyloid-B-induced neuronal dysfunction in Alzheimer's disease: from synapses toward neural networks," Nature Neuroscience, vol. 13, no. 7, pp. 812-818, 2010.

[3] J. Sepulcre, M. R. Sabuncu, A. Becker, R. Sperling, and K. A. Johnson, "In vivo characterization of the early states of the amyloid-beta network," Brain, vol. 136, no. 7, pp. 2239-2252, 2013.

[4] W. W. Seeley, R. K. Crawford, J. Zhou, B. L. Miller, and M. D. Greicius, "Neurodegenerative diseases target large-scale human brain networks," Neuron, vol. 62, no. 1, pp. 42-52, 2009.

[5] M. D. Greicius, G. Srivastava, A. L. Reiss, and V. Menon, "Default-mode network activity distinguishes Alzheimer's disease from healthy aging: evidence from functional MRI," Proceedings of the National Acadamy of Sciences of the United States of America, vol. 101, no. 13, pp. 4637-4642, 2004.

[6] M. Montembeault, I. Rouleau, J.-S. Provost, and S. M. Brambati, "Altered gray matter structural covariance networks in early stages of alzheimer's disease," Cerebral Cortex, vol. 26, no. 6, pp. 2650-2662, 2016.

[7] R. L. Buckner, A. Z. Snyder, B. J. Shannon et al., "Molecular, structural, and functional characterization of Alzheimer's disease: evidence for a relationship between default activity, amyloid, and memory," The Journal of Neuroscience, vol. 25, no. 34, pp. 7709-7717, 2005.

[8] C. Sorg, V. Riedl, and M. Mühlau, "Selective changes of restingstate networks in individuals at risk for Alzheimer's disease," Proceedings of the National Acadamy of Sciences of the United States of America, vol. 104, no. 47, pp. 18760-18768, 2007.

[9] C. R. Jack, D. S. Knopman, W. J. Jagust et al., “Tracking pathophysiological processes in Alzheimer's disease: an updated hypothetical model of dynamic biomarkers," The Lancet Neurology, vol. 12, no. 2, pp. 207-216, 2013.

[10] H.-J. Li, X.-H. Hou, H.-H. Liu, C.-L. Yue, Y. He, and X.-N. Zuo, "Toward systems neuroscience in mild cognitive impairment and Alzheimer's disease: a meta-analysis of 75 fMRI studies," Human Brain Mapping, vol. 36, no. 3, pp. 1217-1232, 2015.

[11] K. Supekar, V. Menon, D. Rubin, M. Musen, and M. D. Greicius, "Network analysis of intrinsic functional brain connectivity in Alzheimer's disease," PLoS Computational Biology, vol. 4, no. 6, Article ID e1000100, 2008.

[12] A. Di Martino, A. Scheres, D. S. Margulies et al., "Functional connectivity of human striatum: a resting state fMRI study," Cerebral Cortex, vol. 18, no. 12, pp. 2735-2747, 2008.

[13] L. C. Walker, M. I. Diamond, K. E. Duff, and B. T. Hyman, "Mechanisms of protein seeding in neurodegenerative diseases," JAMA Neurology, vol. 70, no. 3, pp. 304-310, 2013.

[14] D. T. Jones, D. S. Knopman, J. L. Gunter et al., "Cascading network failure across the Alzheimer's disease spectrum," Brain, vol. 139, no. 2, pp. 547-562, 2016.

[15] S. V. Buldyrev, R. Parshani, G. Paul, H. E. Stanley, and S. Havlin, "Catastrophic cascade of failures in interdependent networks," Nature, vol. 464, no. 7291, pp. 1025-1028, 2010.

[16] W. W. Seeley, V. Menon, A. F. Schatzberg et al., "Dissociable intrinsic connectivity networks for salience processing and executive control," The Journal of Neuroscience, vol. 27, no. 9, pp. 2349-2356, 2007.

[17] M. S. Albert, S. T. DeKosky, D. Dickson et al., "The diagnosis of mild cognitive impairment due to Alzheimer's disease: recommendations from the National Institute on AgingAlzheimer's Association workgroups on diagnostic guidelines for Alzheimer's disease," Alzheimer's \& Dementia, vol. 7, no. 3, pp. 270-279, 2011. 
[18] G. M. McKhann, D. S. Knopman, H. Chertkow et al., “The diagnosis of dementia due to Alzheimer's disease: Recommendations from the National Institute on Aging-Alzheimer's Association workgroups on diagnostic guidelines for Alzheimer's disease," Alzheimer's \& Dementia, vol. 7, no. 3, pp. 263-269, 2011.

[19] Y.-T. Chang, C.-W. Huang, N.-C. Chen et al., "Hippocampal amyloid burden with downstream fusiform gyrus atrophy correlate with face matching task scores in early stage Alzheimer's disease," Frontiers in Aging Neuroscience, vol. 8, 2016.

[20] W. G. Rosen, R. D. Terry, P. A. Fuld, R. Katzman, and A. Peck, "Pathological verification of ischemic score in differentiation of dementias," Annals of Neurology, vol. 7, no. 5, pp. 486-488, 1980.

[21] K.-L. Huang, K.-J. Lin, I.-T. Hsiao et al., "Regional amyloid deposition in amnestic mild cognitive impairment and Alzheimer's disease evaluated by $\left[{ }^{18} \mathrm{~F}\right] \mathrm{AV}-45$ positron emission tomography in Chinese population," PLOS ONE, vol. 8, no. 3, Article ID e58974, 2013.

[22] X. W. Song, Z. Y. Dong, X. Y. Long et al., "REST: a Toolkit for resting-state functional magnetic resonance imaging data processing," PLoS ONE, vol. 6, no. 9, Article ID e25031, 2011.

[23] M. Brendel, M. Högenauer, A. Delker et al., "Improved longitudinal $[18 \mathrm{~F}]-\mathrm{AV} 45$ amyloid PET by white matter reference and VOI-based partial volume effect correction," NeuroImage, vol. 108, pp. 450-459, 2015.

[24] D. R. Gaser, "CAT-A computational anatomy toolbox forthe analysis of structural MRI data," Human Brain Mapping, vol. 2016, 2016.

[25] S. Whitfield-Gabrieli and A. Nieto-Castanon, "Conn: a functional connectivity toolbox for correlated and anticorrelated brain networks," Brain Connectivity, vol. 2, no. 3, pp. 125-141, 2012.

[26] Y. Behzadi, K. Restom, J. Liau, and T. T. Liu, "A component based noise correction method (CompCor) for BOLD and perfusion based fMRI," NeuroImage, vol. 37, no. 1, pp. 90-101, 2007.

[27] M. F. Folstein, S. E. Folstein, and P. R. McHugh, “"Mini mental state". A practical method for grading the cognitive state of patients for the clinician," Journal of Psychiatric Research, vol. 12, no. 3, pp. 189-198, 1975.

[28] C. C. Chang, J. H. Kramer, K. N. Lin et al., "Validating the chinese version of the verbal learning test for screening alzheimer's disease," Journal of the International Neuropsychological Society, vol. 16, no. 2, pp. 244-251, 2010.

[29] E. K. Warrington and M. James, The Visual Object and Space Perception Battery, Thames Valley Test Company, Bury St Edmunds, England, 1991.

[30] K. B. Boone, "The boston qualitative scoring system for the reyosterrieth complex figure," Journal of Clinical and Experimental Neuropsychology, vol. 22, no. 3, pp. 430-432, 2000.

[31] H. Amieva, S. Lafont, I. Rouch-Leroyer et al., "Evidencing inhibitory deficits in Alzheimer's disease through interference effects and shifting disabilities in the Stroop test," Archives of Clinical Neuropsychology, vol. 19, no. 6, pp. 791-803, 2004.

[32] R. M. Reitan, "The relation of the Trail Making Test to organic brain damage," Journal of Consulting and Clinical Psychology, vol. 19, no. 5, pp. 393-394, 1955.

[33] R. B. Postuma and A. Dagher, "Basal ganglia functional connectivity based on a meta-analysis of 126 positron emission tomography and functional magnetic resonance imaging publications," Cerebral Cortex, vol. 16, no. 10, pp. 1508-1521, 2006.

[34] R. Petersen, Ed., Mild Cognitive Impairment: Aging to Alzheimer's Disease, Oxford University Press, New York, NY, USA, 2003.
[35] T. A. Crowell, C. A. Luis, D. E. Cox, and M. Mullan, "Neuropsychological comparison of Alzheimer's disease and dementia with Lewy bodies," Dementia and Geriatric Cognitive Disorders, vol. 23, no. 2, pp. 120-125, 2007.

[36] M. A. Lambon Ralph, K. Patterson, N. Graham, K. Dawson, and J. R. Hodges, "Homogeneity and heterogeneity in mild cognitive impairment and Alzheimer's disease: A cross-sectional and longitudinal study of 55 cases," Brain, vol. 126, no. 11, pp. 23502362, 2003.

[37] K. Takehara-Nishiuchi, "Entorhinal cortex and consolidated memory," Neuroscience Research, vol. 84, pp. 27-33, 2014.

[38] J. Ferbinteanu, "Contributions of hippocampus and striatum to memory-guided behavior depend on past experience," The Journal of Neuroscience, vol. 36, no. 24, pp. 6459-6470, 2016.

[39] J. D. Riley, S. Moore, S. C. Cramer, and J. J. Lin, "Caudate atrophy and impaired frontostriatal connections are linked to executive dysfunction in temporal lobe epilepsy," Epilepsy \& Behavior, vol. 21, no. 1, pp. 80-87, 2011.

[40] M. Marquie, J. J. Locascio, D. M. Rentz et al., "Striatal and extrastriatal dopamine transporter levels relate to cognition in Lewy body diseases: An $11 \mathrm{C}$ altropane positron emission tomography study," Alzheimer's Research \& Therapy, vol. 6, no. 8, 2014.

[41] A. Alexander-Bloch, J. N. Giedd, and E. Bullmore, "Imaging structural co-variance between human brain regions," Nature Reviews Neuroscience, vol. 14, no. 5, pp. 322-336, 2013.

[42] E. C. Mormino, A. Smiljic, A. O. Hayenga et al., "Relationships between beta-amyloid and functional connectivity in different components of the default mode network in aging," Cerebral Cortex, vol. 21, no. 10, pp. 2399-2407, 2011.

[43] N. Myers, L. Pasquini, J. Göttler et al., "Within-patient correspondence of amyloid- $\beta$ and intrinsic network connectivity in Alzheimer's disease," Brain, vol. 137, no. 7, pp. 2052-2064, 2014.

[44] C. R. Jack, P. Vemuri, H. J. Wiste, S. D. Weigand, T. G. Lesnick, and V. Lowe, "Shapes of the trajectories of five major biomarkers of alzheimer's disease," Archives of Neurology, vol. 69, no. 7, p. 12, 2012.

[45] C. R. Jack, H. J. Wiste, T. G. Lesnick et al., "Brain beta-amyloid load approaches a plateau," Neurology, vol. 80, no. 10, pp. 890896, 2013.

[46] C. D. Hacker, J. S. Perlmutter, S. R. Criswell, B. M. Ances, and A. Z. Snyder, "Resting state functional connectivity of the striatum in Parkinson's disease," Brain, vol. 135, no. 12, pp. 3699-3711, 2012.

[47] O. Monchi, M. Petrides, B. Mejia-Constain, and A. P. Strafella, "Cortical activity in Parkinson's disease during executive processing depends on striatal involvement," Brain, vol. 130, no. 1, pp. 233-244, 2007.

[48] M. Carbon and R. M. Marie, "Functional imaging of cognition in Parkinson's disease," Current Opinion in Neurology, vol. 16, pp. 475-480, 2003.

[49] M. E. Kalaitzakis, M. B. Graeber, S. M. Gentleman, and R. K. Pearce, "Striatal $\beta$-amyloid deposition in parkinson disease with dementia," Journal of Neuropathology \& Experimental Neurology, vol. 67, no. 2, pp. 155-161, 2008.

[50] W. E. Klunk, J. C. Price, C. A. Mathis et al., "Amyloid deposition begins in the striatum of presenilin-1 mutation carriers from two unrelated pedigrees," The Journal of Neuroscience, vol. 27, no. 23, pp. 6174-6184, 2007.

[51] M. J. Grothe, H. Barthel, J. Sepulcre, M. Dyrba, O. Sabri, and S. J. Teipel, "In vivo staging of regional amyloid deposition," Neurology, vol. 89, no. 20, pp. 2031-2038, 2017. 


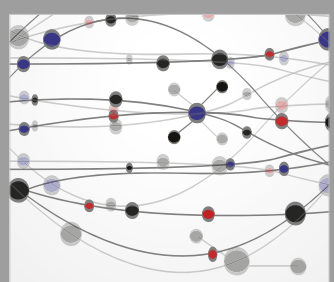

The Scientific World Journal
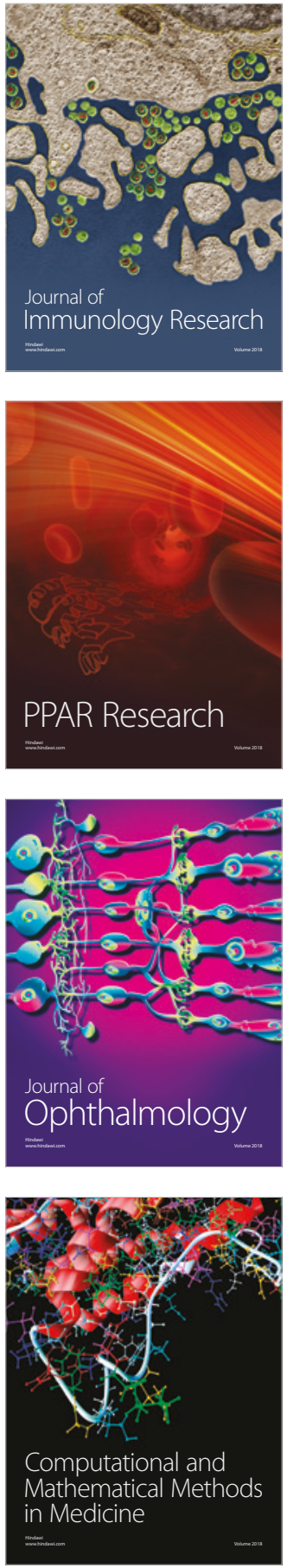

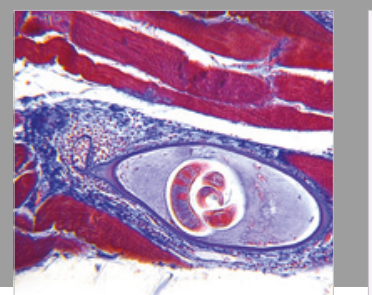

Gastroenterology Research and Practice

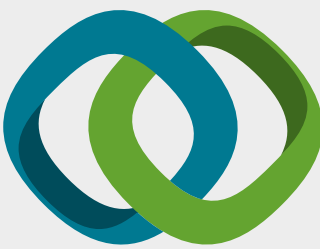

\section{Hindawi}

Submit your manuscripts at

www.hindawi.com
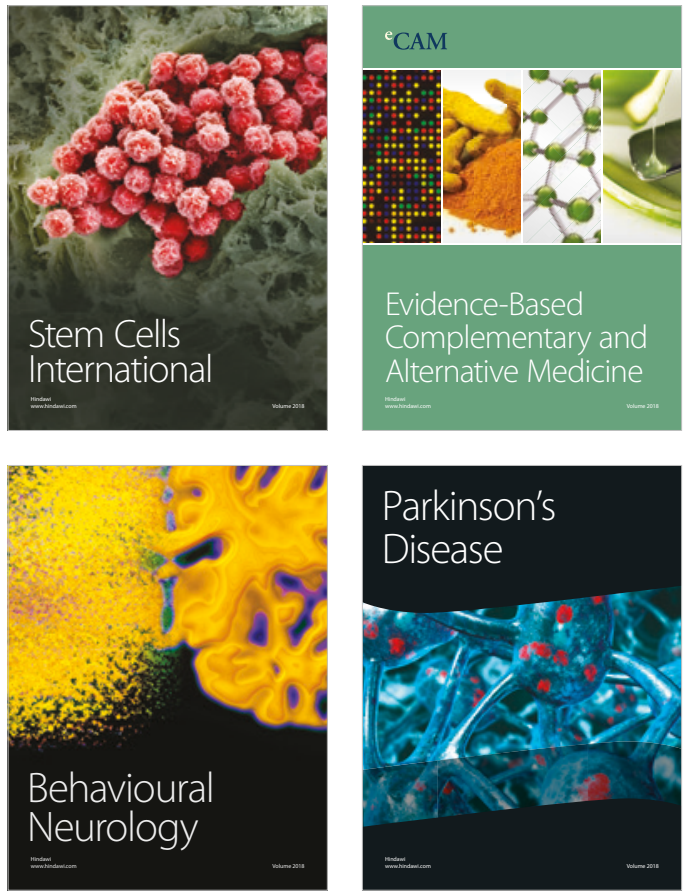

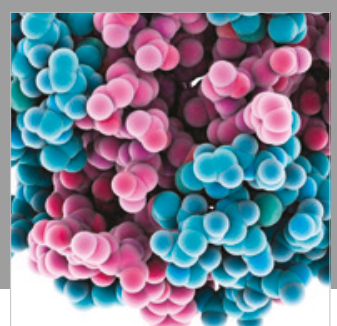

ournal of

Diabetes Research

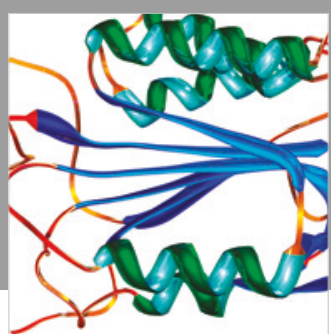

Disease Markers
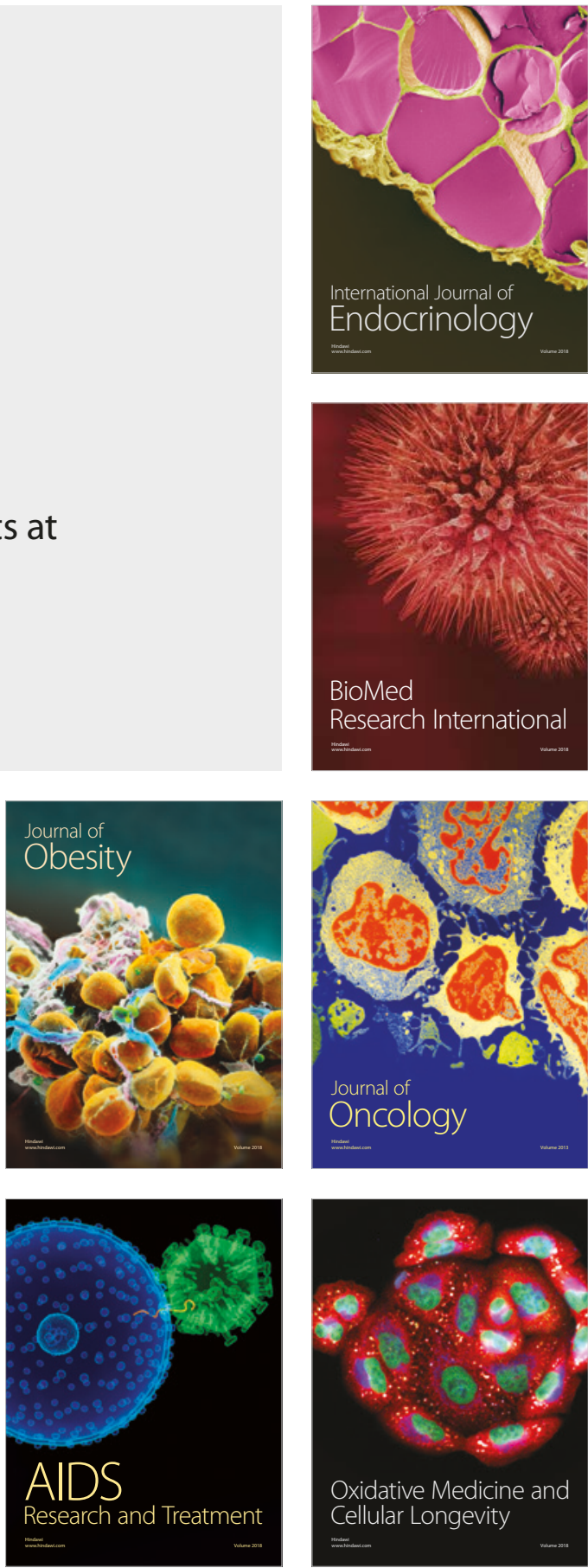